\title{
Self-reported pregnancy-related health problems and self-rated health status in Rwandan women postpartum: a population-based cross-sectional study
}

Jean Paul S. Semasaka ${ }^{1,2^{*}}$, Gunilla Krantz ${ }^{3}$, Manasse Nzayirambaho², Cyprien Munyanshongore ${ }^{2}$, Kristina Edvardsson ${ }^{1,4}$ and Ingrid Mogren ${ }^{1}$

\begin{abstract}
Background: Self-rated health status (SRH) can be used as a predictor of morbidity and mortality. Postpartum selfrated health has been used to estimate maternal morbidity and postpartum problems. Reproductive history factors are associated with poor self-rated health postpartum. This study investigated prevalence of self-reported health problems during first, second, and third trimesters of pregnancy, delivery, and postpartum. In addition, this study investigated SRH in Rwandan women up to 13 months from partus.

Methods: This population-based, cross-sectional study collected data in 2014 using structured interviews ( $N=921)$. Univariable analysis was used to identify variables that were associated with poor self-rated health status (poorSRH). Logistic regression analyses were performed to identify factors associated with poor-SRH at one day, one week, and one month postpartum and at the time of the interview.

Results: Mean time between latest delivery and the time of interview was 7.1 months. Prevalence of anaemia, hypertension, diabetes mellitus during pregnancy, and severe bleeding during pregnancy and labour were 15.0, 4.9, 2.4 , and $3.7 \%$, respectively. The prevalence of poor-SRH was $32.2 \%$ at one day postpartum, $7.8 \%$ at one month, and $11.7 \%$ at time of the interview. Hypertension during pregnancy and significant postpartum haemorrhage were associated with poor-SRH at one day and one week postpartum. Severe bleeding during pregnancy and labour were associated with poor-SRH at one week and one month postpartum. Infection and anaemia during pregnancy were associated with poor-SRH at one month postpartum and at the time of interview. The Kaplan-Meier curves illustrate restitution of health for most women during the study period.

Conclusions: This population-based study reports a high prevalence of poor SRH status among Rwandan women in the early postpartum period. Identified factors associated with poor-SRH were severe bleeding, hypertension, infection, and anaemia during pregnancy and postpartum haemorrhage. These factors may be prevented or reduced by providing more frequent and specific attention during pregnancy and by providing timely measures that address complications during delivery, including adequate postpartum care.
\end{abstract}

Keywords: Self-rated health status, Pregnancy, Rwanda, Reproductive history, Cross-sectional study, Pregnancyrelated complications

\footnotetext{
* Correspondence: jeanpaul.semasaka.sengoma@umu.se

'Department of Clinical Sciences, Obstetrics and Gynecology, Umeå

University, Umeå, Sweden

${ }^{2}$ University of Rwanda College of Medicine and Health Sciences School of

Public Health, Kigali, Rwanda

Full list of author information is available at the end of the article
} 


\section{Background}

Self-rated health status (SRH) is recognised as a global measure of estimation of quality of life and a good predictor of morbidity and mortality [1-3]. SRH uses subjective questions, to ask how people perceive their health status. These questions summarize the participants' health status, including physical, psychological, and social dimensions that are not easily accessible to an objective observer $[4,5]$.

In 2012, an estimated 213 million pregnancies occured in the world and more than 1.5 million of these pregnancies had pregnancy-related complications $[6,7]$. The most common pregnancy-related complications are postpartum haemorrhage, hypertensive disorders (including preeclampia and eclampsia), sepsis, obstructive or prolonged labour, and spontaneous abortion [8]. Health problems such as headache, low back pain, anxiety, depression, urinary incontinence, and feacal incontinence may remain after childbirth [9-12]. There is poor documentation globally about the extent and the nature of women's postpartum health status [13-16]. Poor self-rated health status has been found to be associated with reproductive history factors such as being a single mother, higher parity, delivery by caesarean section, and young age $[10,17,18]$.

In 2013, an estimated 323,197 women gave birth in Rwanda, and $14.7 \%$ of these women were delivered by caesarean section $[19,20]$. Rwanda, like other low income countries, has made sincere efforts during the last decade to decrease maternal mortality and morbidity [21]. In Rwanda, from 2010 to 2015, the four recommended antenatal care (ANC) visits by the World Health Organization (WHO) increased from 35 to $44.3 \%$, with the incease number of women having the first ANC visit during the first trimester of pregnancy from 38 to $56.3 \%$. Skilled provider assistance during delivery increased from 69 to $91 \%$, and uptake of postnatal care from 18 to $43 \%$. Moreover, maternal mortality ratio was estimated to have decreased from 476 to 210 per 100000 live births during the same period [21-23].

\section{Rationale of the study}

There is insufficient information on rates of pregnancyrelated complications in Rwanda. In addition, health status postpartum in Rwandan women is not well described. This study aims to fill the knowledge gap in this area and to serve as a base for policy-makers in their decision-making in order to improve maternal and foetal health and women's health postpartum.

\section{Aims}

In a population-based sample, this study investigated the self-rated overall health status in relation to reproductive history within 13 months from partus. Specific aims to investigate were: $i$ ) prevalence of self-reported health problems during first, second, and third trimesters of pregnancy, delivery, and postpartum ii) self-rated overall health status and its determinants at one day, one week, and one month postpartum and at the time of the interview.

This study is part of the Maternal Health Research Programme (MaTHeR) undertaken by the University of Rwanda in collaboration with University of Gothenburg and Umeå University in Sweden.

\section{Methods}

\section{The study setting}

The Rwandan health system is decentralised with the community level as the first level of maternal health service provision. There are three community health workers (CHWs) in each village including Animatrice de Santé Maternelle (ASM), a CHW who is exclusively in charge of maternal health services $[24,25]$. The secondary level of maternal health services is the health center. The majority of women with uncomplicated pregnancies deliver at health centres, while complicated cases are referred to the district hospital level or to the referral hospitals according to the severity of the pregnancyrelated problem [25].

This study was conducted in two locations in Rwanda: the city of Kigali and the Northern Province. Kigali includes urban, semi-urban, and rural areas with a total population of 1,135,428 and 1910 villages. The Northern Province includes mainly semi-urban and rural areas with a population of $1,729,927$ and 2881 villages [26].

\section{Methodology of the study and study participants Sampling}

This study applied a cross-sectional study design. A sample size of 922 women was calculated based on the estimated prevalence of pregnancy-related hypertension (10\%) [8] with an absolute precision of $5 \%$, a $10 \%$ possibility of non-responses, and a design effect of 1.5. A sampling frame prepared by the National Institute of Statistics of Rwanda (NISR) for the Rwandan general population and household census conducted in 2012 was used. This sampling frame is a complete list of villages covering the Northern Province and Kigali. From this sampling frame, 48 villages $(1 \%)$ were randomly selected. Eligible participants were women who gave birth within 13 months before data collection and who were identified with the assistance of the ASM. The final sample consisted of 921 as one contact did not participate, giving a response rate of $99.9 \%$.

\section{Questionnaire}

A questionnaire was developed by the research team that included questions about sociodemographic and psychosocial factors, pregnancies before the latest pregnancy, 
latest pregnancy, latest delivery, and postpartum situation. Sociodemographic background characteristics included age, marital status, and educational level. The majority of questions in the questionnaire were closed ended questions with a fixed number of response alternatives and Likert-type scale questions. For five questions in this study, the respondents were also given the possibility to give additional written comments. The questionnaire included questions about previous pregnancies as well as more detailed questions related to health problems during latest pregnancy, latest delivery, and postpartum period. Participants were also asked to report their SRH at one day postpartum, one week postpartum, one month postpartum, and at the time of the interview. First written in English, the questionnaire was translated into Kinyarwanda. Thereafter, the questionnaire was tested in a pilot study. The pilot study included 36 women from a village neighbouring a selected village of the study. All 36 questionnaires were completed, and apart from adjustments of the wording of some questions, no major revision of the questionnaire was needed after the pilot study.

\section{Data collection procedures}

All data were collected by a group of 12 female experienced interviewers (nurses, midwives, and clinical psychologists) through individual structured interviews to secure completeness of data. Before conducting the interviews, the interviewers participated in a five-day training. The data collection was performed between July 2014 and August 2014. At the end of each day, during the first three days of data collection, at least one participant per village was re-interviewed in order to check the completeness of the questionnaires and the accuracy of data collected. After the primary data entry, the information from 100 questionnaires, each including the 117 variables used in this study, were re-registered to check the accuracy of the first data entry. In total, 30 errors were detected which corresponds to an error rate of $0.25 \%(30 / 11700)$. The erroneous data were thereafter corrected.

\section{Dependent variables}

The participants retrospectively reported their SRH four times postpartum: one day, one week, one month, and at the time of the interview. There were five available response options: very good, good, neither good nor poor, poor, and very poor. In a subpart of analysis, the variable was dichotomised into two categories labelled good health status (good-SRH) for those who rated their health as very good or good, and poor health status (poor-SRH) for those who rated their health status as very poor or poor or neither good nor poor.

\section{Independent variables}

Sociodemographic and psychosocial variables were analyzed as independent variables. Women's age was a continuous numerical variable that was divided into five age categories: less than 25 years, 25-29 years, 30-34 years, 35-39 years, and more than 40 years. Marital status included married, cohabiting, separated or divorced, widowed, and unmarried or single. Age at marriage was a continuous numerical variable that was categorized into less than 20 years, $21-30$ years, and more than 30 years. Woman's education was a combination of two primary variables: having attended school (yes or no) and educational level. Educational level included primary level not complete, primary level completed, vocational training, secondary level senior 1-4, secondary school senior 5-6, tertiary level, and a do not know option. The two variables were grouped into four categories: no education, completed primary level, completed secondary school or vocational training, and tertiary university level. Woman's occupation included student, unskilled worker, skilled worker, civil servant, not employed, and other employment. Place of delivery included delivery at home, on the way to the health facility, at health post/dispensary, at the health centre, at district/provincial hospital, at referral hospital, at a private clinic, at any other health facility, and at any other place. Mode of delivery included delivered vaginally without instruments, vaginally with forceps, vaginally with vacuum extraction, planned caesarean section, and emergency caesarean section. Health insurance included the categories no health insurance, community health-based health insurance, public health insurance, and private health insurance. A new variable handicapping complication was created for women who reported either fistula, urinary incontinence, or fecal incontinence. First trimester was defined as the first three months of the pregnancy. Second trimester was defined as four to six months of pregnancy. Third trimester was defined as seven months or more. Variables about main health problems during pregnancy and delivery were for each variable collected for the first, second, and the third trimesters. Thereafter, each variable - diabetes mellitus, anaemia, and infections (composed essentially by urinary infection) during the first, the second, the third trimester - were combined to become diabetes mellitus during pregnancy, anaemia during pregnancy, and infections during pregnancy. Significant vaginal blood loss within $24 \mathrm{~h}$ after delivery and significant vaginal blood loss within the first weeks after delivery were combined into significant blood loss after delivery. Further categorization of variables are presented in first three tables.

\section{Statistical analysis}

Prevalence rates were calculated for description of different variables related to pregnancy, delivery, and postpartum. 
The study identified factors related to poor-SRH postpartum using univariable logistic regression analysis. Variables that were statistically significantly associated with poorSRH status were considered for the final logistic regression model. Finally, a multivariable logistic regression model was built that calculated odds ratios (OR) and their $95 \%$ confidence intervals (CI). In the multivariable model, forward stepwise regression was used. All statistically significant variables in univariable analyses were entered one by one to identify factors that had a relationship with poorSRH at one day, one week, and one month postpartum and at time of the interview, keeping in the final model only factors that were statistically significant $(p<0.05)$. All multivariable models included number of births, women's age, mode of delivery, and marital status for theoretical reasons. A Kaplan-Meier analysis using the curve "One minus survival" with a log rank test was constructed to illustrate the time-dependent self-rated poor health status in relation to anaemia during pregnancy, women's level of education, and significant postpartum haemorrhage. This analysis was done to illustrate improvement of health status in women who reported low level of education, anaemia during pregnancy, or significant postpartum haemorrhage compared to those without these factors during the follow-up period. These analyses only included women rating their health as poor-SRH at one day postpartum. Thus, the Kaplan-Meier analyses included a sub-category of 296 participants. The time end point for each participant was the time of the interview. All analyses were performed in SPSS version 22.

\section{Results}

\section{Sociodemographic and reproductive history} characteristics

A total of 921 women aged 15 to 46 years with a mean age of 27.9 years were enrolled in this study. The average period between the date of the interview and the date of the latest delivery was 7.1 months (range: 1.4-14.3 months). The distribution of proportions in relation to the postpartum follow-up time were $35.3 \%$ for $1-5$ months, $31.6 \%$ for 5.1-9 months, and $33.1 \%$ for 9.1-14.3 months. Frequencies and percentages of sociodemographic and reproductive history characteristics of participants are presented in Table 1. Married or cohabiting women constituted $84.1 \%$ of participants, and single or unmarried women corresponded to $13.4 \%$ of the sample. Primiparus mothers represented $30.7 \%$ of the participants and multiparous women with more than four births represented $24.2 \%$ of the participants. A large majority of participants (87.6\%) reported a normal vaginal delivery at a health facility, and $5.1 \%$ reported delivery at home or on the way to a health facility.

Health problems during pregnancy and delivery period Prevalence of self-reported, pregnancy-related health problems during first, second, and third trimesters of pregnancy are presented in Table 2. Self-reported prevalence of anaemia, hypertension, diabetes mellitus, and severe bleeding during pregnancy were 15.0, 4.9, 2.2, and $2.0 \%$, respectively, and $15.7 \%$ reported being transferred pre-delivery from a health center to a district hospital or to a referral hospital due to health problems. Abnormal foetal position was reported in $3.9 \%$ of cases.

\section{Health problems during postpartum period}

A total of $8.5 \%$ of participants reported significant postpartum haemorrhage immediately after delivery or within $24 \mathrm{~h}$, and $4.7 \%$ reported significant postpartum haemorrhage during their first week postpartum. At the time of the interview, the prevalence of almost daily or weekly irritability, anxiety, and depression was $6.5 \%, 22.6 \%$, and $14.8 \%$, respectively. A total of $2.0 \%$ of the participants reported having developed vaginal fistula, $0.4 \%$ reported fecal incontinence, $2.4 \%$ reported urinary incontinence, and $3.3 \%$ reported problems related to an episiotomy during most recent delivery. Generalized fatigue almost daily or weekly was reported by $17.3 \%$, severe headache by $21.7 \%$, and low back pain by $26.4 \%$.

\section{Self-rated overall health status postpartum}

The proportion of women who rated their overall health status as very poor or poor decreased during the study period (Fig. 1). Almost a third of participants (32.2 \%) reported poor-SRH at one day, but only $11.7 \%$ were still reporting poor-SRH at the time of the interview. The Kaplan-Meier curves illustrate that women with low level of education, women with anaemia during pregnancy, and women with significant postpartum haemorrhage reported poorer health status during the early part of the postpartum period, compared to women with none of these conditions. However, the log rank test revealed that for the entire follow-up period there was no statistically significant difference between groups for self-rated overall health status in relation to level of education $(p=0.863)$, anaemia during pregnancy $(p=0.463)$, and significant postpartum haemorrhage $(p=0.401)$. For those women who were followed-up to around one year postpartum, there was no significant difference in overall health status between women characterized by different categories of exposures (Figs. 2, 3, and 4). Thus, women had in general regained a good health status.

Background characteristics and pregnancy outcomes and their associations with self-rated overall health status at different time points postpartum Self-rated overall health status at one day postpartum Reproductive factors that were associated with poor-SRH at one day postpartum in univariable logistic regression analyses are presented in Table 3. In a multivariable logistic regression model, the following reproductive factors were 
Table 1 Socio-demographic and reproductive history background characteristics of participants

\begin{tabular}{|c|}
\hline Variables \\
\hline Women's mean age \\
\hline Women's mean number of years of education \\
\hline Husband's mean age \\
\hline $\begin{array}{l}\text { Husband's mean number of years of education } \\
\text { mean }\end{array}$ \\
\hline Women's age (years) \\
\hline$<25$ \\
\hline $25-29$ \\
\hline $30-34$ \\
\hline $35-39$ \\
\hline$\geq 40$ \\
\hline Marital status \\
\hline Single or unmarried \\
\hline Widow \\
\hline Separated or divorced \\
\hline Cohabiting \\
\hline Married \\
\hline Age at marriage (years) \\
\hline$<20$ \\
\hline $21-30$ \\
\hline$>30$ \\
\hline Woman's education \\
\hline No education \\
\hline Completed primary level \\
\hline Secondary school and vocational training \\
\hline Tertiary, university level \\
\hline Woman's occupation \\
\hline Student \\
\hline Non skilled worker \\
\hline Skilled worker \\
\hline Civil servant \\
\hline Not employed \\
\hline Other employment \\
\hline Place of delivery \\
\hline At home or on the way to the health facility \\
\hline At public health facility \\
\hline At private or any other health facility \\
\hline Mode of delivery \\
\hline Vaginal without instruments ${ }^{b}$ \\
\hline Vaginal with forceps \\
\hline Vaginal with vacuum extraction \\
\hline Planned caesarean section \\
\hline Emergency caesarean section \\
\hline
\end{tabular}

920

277

212

98

38

920

123

13

10

292

482

804

339

447

18

921

97

608

178

38

920

17

529

\section{7}

862

10

913

798

4

0

33

78
Table 1 Socio-demographic and reproductive history background characteristics of participants (Continued)

\begin{tabular}{ll} 
Mean age (years) & $S^{\mathrm{a}}$ \\
\hline 27.89 & 6.02 \\
5.80 & 3.10 \\
32.55 & 7.74 \\
6.14 & 3.16
\end{tabular}

Nu

889

96.5

273

30.7

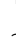

3

32

221

3.6

4

24.9

$>4$

16.6

Discharge time (days)

24.2

N $\%$

99.9

32.0

$<3$

3

4-7

$>7$

Health insurance

148

215

899

474

97.6

No insurance

Community health based insurance

Public insurance (RAMA, MMI, MIS/UR) and other private

273

52.7

30.4

119

13.2

33

3.7

920

99.9

99.9

13.4

1.4

1.1

ANC visits

188

20.4

686

74.5

47

5.1

31.7

Yes

915

99.3

52.4

87.3

36.8

No

Number of ANC visits

$1-3$

915

0.5

99.3

$\geq 4$

54.3

45.7

Religion

99.8

Catholicism

Protestantism

46.6

Adventist

36.3

Islam

8.4

1.7

Other religion

6.3

No religion

0.7

Husband's age group (years)

84.0

$$
<25
$$

8.5

25-29

26.1

30-34

21.1

35-39

12.9

40-44

9.3

$\geq 45$

6.1
99.8

5.1

93.6

1.1

99.1

86.6

0.4

0

3.6

8.5 
Table 1 Socio-demographic and reproductive history background characteristics of participants (Continued)

\begin{tabular}{lll}
\hline Husband's education & 791 & 85.0 \\
No education & 100 & 10.9 \\
Completed primary level & 527 & 57.3 \\
Secondary school and vocational training & 117 & 12.7 \\
Tertiary, university level & 32 & 3.5 \\
Don't know & 15 & 1.6 \\
Household income per month & 914 & 99.2 \\
$<17,500$ RwF & 258 & 28.2 \\
17,500-35,000 RwF & 240 & 26.3 \\
$>35,000$ RwF & 416 & 45.5
\end{tabular}

${ }^{\mathrm{a}} \mathrm{SD}=$ Standard deviation

${ }^{\mathrm{b}}$ Vaginal without instruments = normal delivery without forceps nor vacuum extraction

${ }^{c}$ RAMA = La Rwandaise d'Assurance Maladie, $M M I=$ Military Medical Insurance, MIS/UR = Medical Insurance of University of Rwanda statistically significantly associated with poor-SRH at one day postpartum: caesarean section (reference group (ref.): vaginal delivery with or without instruments), hypertension during pregnancy and delivery (ref.: no hypertension), and significant postpartum haemorrage (ref.: no significant postpartum haemorrage) (Table 4).

\section{Self-rated overall health status at one week postpartum}

The univariable logistic regression analyses revealed several variables statistically significally associated with poorSRH one week postpartum: anaemia during pregnancy $(\mathrm{OR}=1.78, \mathrm{CI}=1.15-2.75$; ref.: no anaemia), caesarean section as mode of delivery $(\mathrm{OR}=2.63, \mathrm{CI}=1.68-4.11$; ref.: vaginal delivery with or without instruments), hypertension during pregnancy and delivery $(\mathrm{OR}=3.41, \mathrm{CI}=$ 1.87-6.24; ref.: no hypertension), severe bleeding during pregnancy and labour $(\mathrm{OR}=3.69, \mathrm{CI}=1.82-7.48$; ref.: no severe bleeding), significant postpartum haemorrage $(\mathrm{OR}=2.60, \mathrm{CI}=1.61-4.21$; ref.: no significant postpartum haemorrage), handicapping complication (i.e., postpartum fistula or fecal and urinary

Table 2 Prevalence of self-reported pregnancy-related problems in 1st, 2nd, and 3rd trimesters ${ }^{\mathrm{a}}$ of pregnancy, and the cumulative prevalence

\begin{tabular}{|c|c|c|c|c|c|c|c|c|}
\hline \multirow[t]{2}{*}{ Variable } & \multicolumn{2}{|c|}{ 1st trimester } & \multicolumn{2}{|c|}{ 2nd trimester } & \multicolumn{2}{|c|}{ 3rd trimester } & \multicolumn{2}{|c|}{ Cumulative prevalence } \\
\hline & $n$ & $\%$ & $n$ & $\%$ & $n$ & $\%$ & $n$ & $\%$ \\
\hline Hypertension & 28 & 3 & 14 & 1.4 & 17 & 1.8 & 45 & 4.9 \\
\hline Convulsions & 18 & 2 & 3 & 0.3 & 5 & 0.5 & 22 & 2.4 \\
\hline Diabetes mellitus & 11 & 1.2 & 10 & 1.1 & 3 & 0.3 & 20 & 2.2 \\
\hline Bad smelling and vaginal discharge & 41 & 4.5 & 47 & 5.1 & 29 & 3.1 & 85 & 9.2 \\
\hline Anaemia & 66 & 7.2 & 81 & 8.8 & 58 & 6.3 & 138 & 15.0 \\
\hline Severe vaginal bleeding & & & 14 & 1.5 & 6 & 0.7 & 18 & 2.0 \\
\hline Severe or continuous headache & & & 59 & 6.4 & 46 & 5.0 & 72 & 7.8 \\
\hline Dimness or blurring vision & & & 99 & 10.9 & 62 & 6.7 & 119 & 12.9 \\
\hline Abdominal pain and severe bleeding & & & 16 & 1.7 & 5 & 0.5 & 177 & 19.2 \\
\hline Vomiting & & & 105 & 11.4 & 44 & 4.8 & 117 & 12.7 \\
\hline Fever & & & 26 & 1.3 & 9 & 1.0 & 29 & 3.1 \\
\hline Leaking of fluid from vagina & & & 54 & 5.9 & 61 & 6.6 & 82 & 8.9 \\
\hline Swollen extremities & & & 55 & 6.0 & 135 & 14.7 & 157 & 17 \\
\hline Preterm premature rupture of membranes & & & 4 & 0.4 & 12 & 1.3 & 16 & 1.7 \\
\hline Diarrhoea & & & 18 & 2.0 & 7 & 0.8 & 25 & 2.7 \\
\hline Baby not moving normally & & & 9 & 1.0 & 5 & 0.5 & 12 & 1.3 \\
\hline Abdominal pain & & & 93 & 10.1 & 93 & 10.1 & 177 & 19.2 \\
\hline Regular and painful uterine contractions & & & 14 & 1.5 & 18 & 2.0 & 29 & 3.1 \\
\hline Infection & & & 12 & 1.3 & 11 & 1.2 & 19 & 2.1 \\
\hline Preterm labour & & & & & 29 & 3.1 & 29 & 3.1 \\
\hline
\end{tabular}

${ }^{\mathrm{a}}$ The first trimester represents the first three months of pregnancy, the second trimester represents four to six months of pregnancy, and the third trimester was defined as seven months or more

${ }^{\mathrm{b}}$ The third trimester does not include events or complications during delivery 


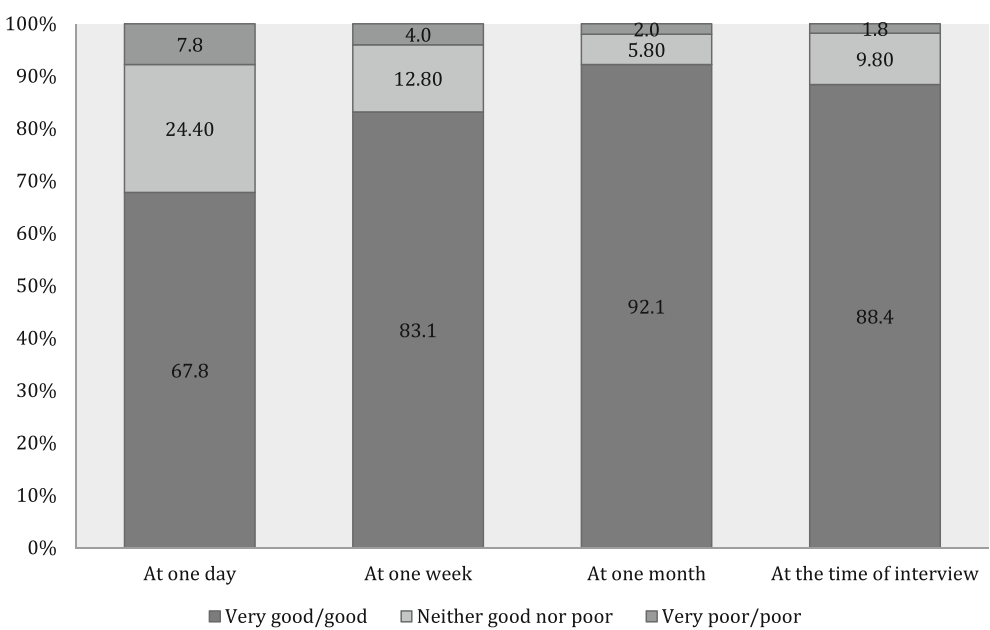

Fig. 1 Self-rated health status postpartum at one day, one week, one month, and at time of interview. Different self-rated health status categories are presented with proportions (\%)

incontinance $) \quad(\mathrm{OR}=3.34, \quad \mathrm{CI}=1.67-6.70 ; \quad$ ref.: $\quad$ no handicapping complication), woman's age less than 25 year $(\mathrm{OR}=1.61, \mathrm{CI}=1.10-2.35$; ref.: age group 25 to 34 years of age), discharge time seven days postpartum $(\mathrm{OR}=4.32, \mathrm{CI}=2.07-9.02$; ref.: discharge time less than 3 days), and convulsions during pregnancy, delivery, and up to one month postpartum $(\mathrm{OR}=2.35$, $\mathrm{CI}=1.12-4.91$; ref.: no convulsions).

In a multivariable logistic regression model $(n=858)$, the following reproductive factors were statistically significantly associated with poor-SRH one week postpartum: caesarean section $(\mathrm{OR}=1.95, \mathrm{CI}=1.08-5.53)$,

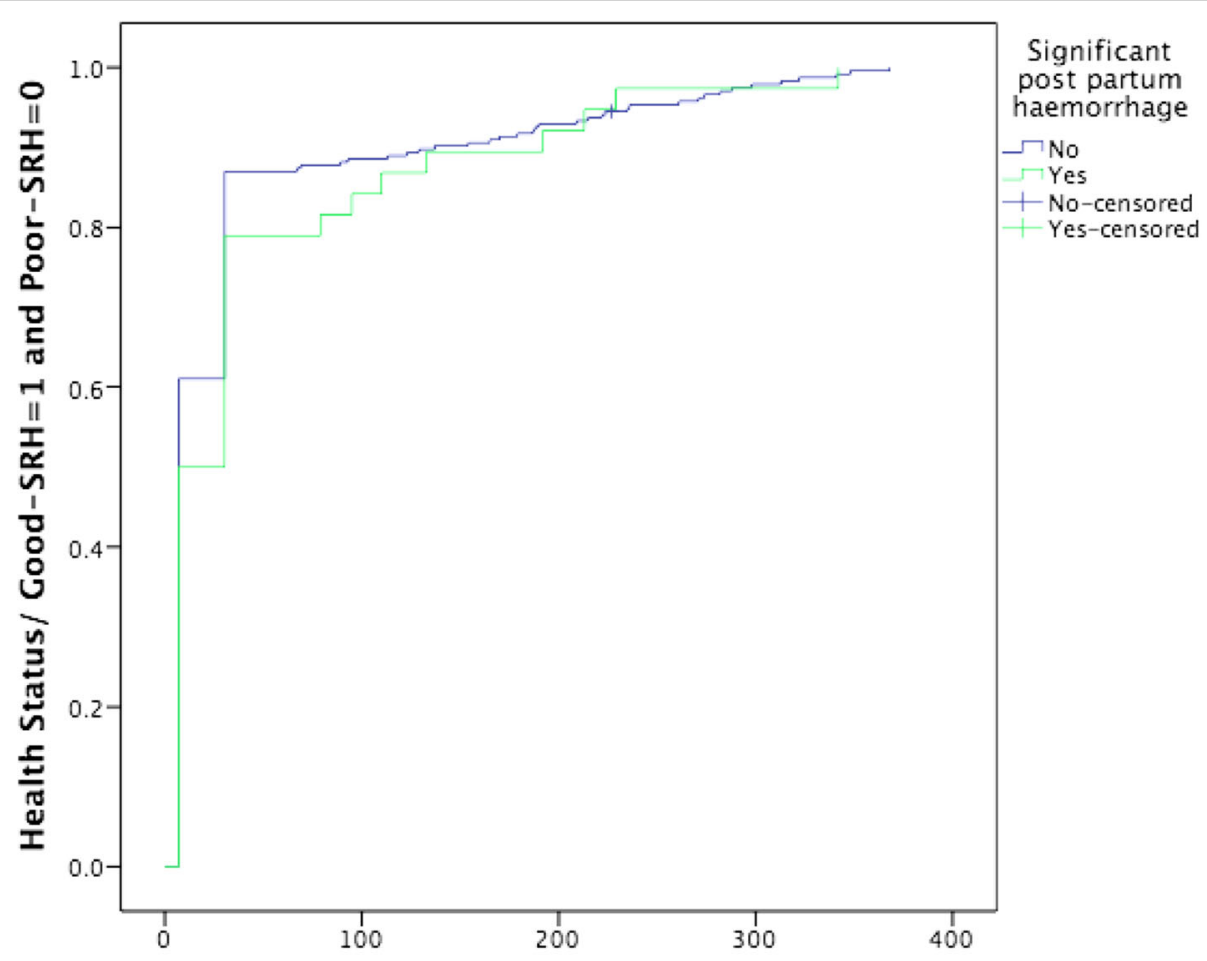

Time for event in days

Fig. 2 Kaplan-Meier curve showing change in health status from poor-SRH to good-SRH by experience of significant post partum haemorrage

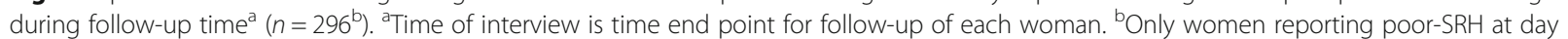
one are included in analysis 


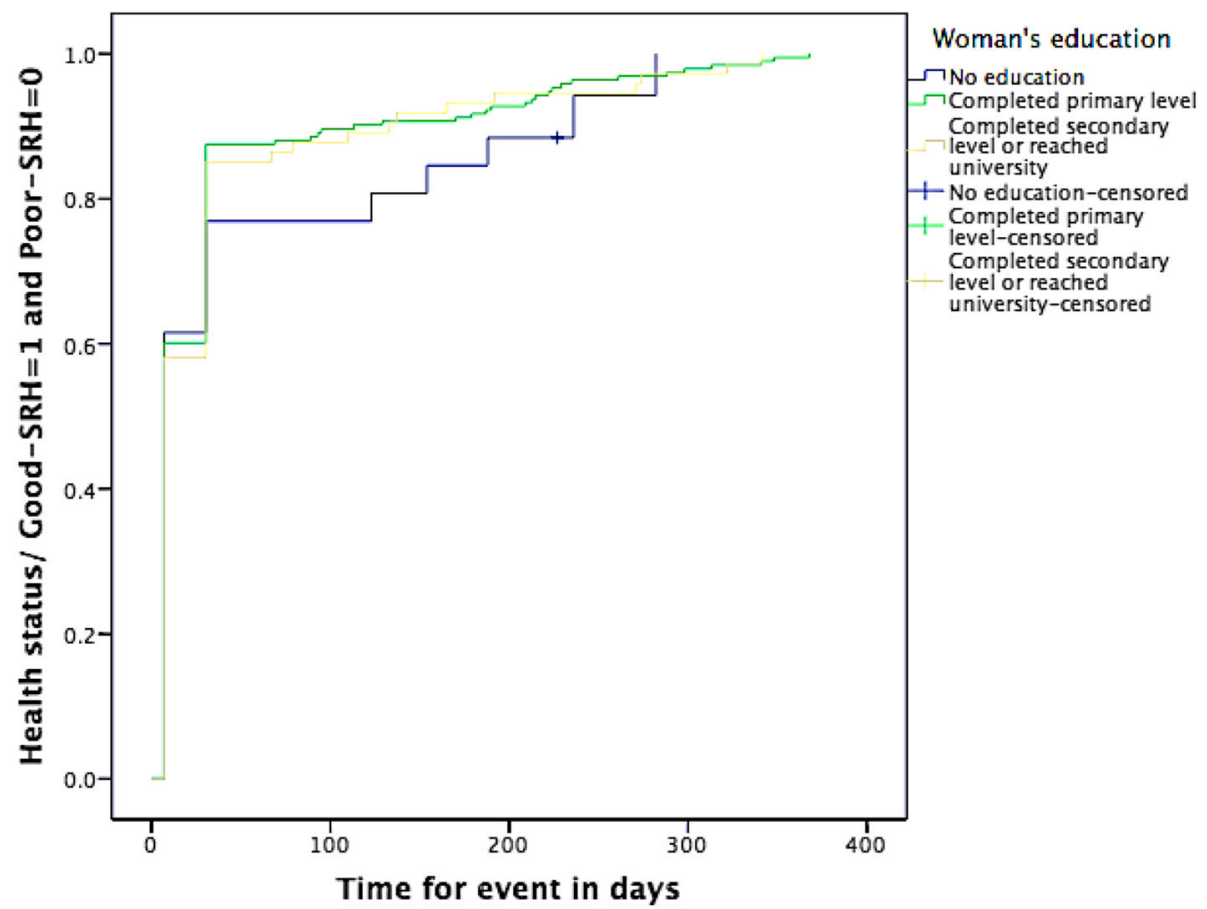

Fig. 3 Kaplan-Meier curve showing change in health status from poor-SRH to good-SRH by educational level during follow-up time ${ }^{\mathrm{a}}\left(n=296^{\mathrm{b}}\right)$. aTime of interview is time end point for follow-up of each woman. ${ }^{b}$ Only women reporting poor-SRH at day one are included in analysis

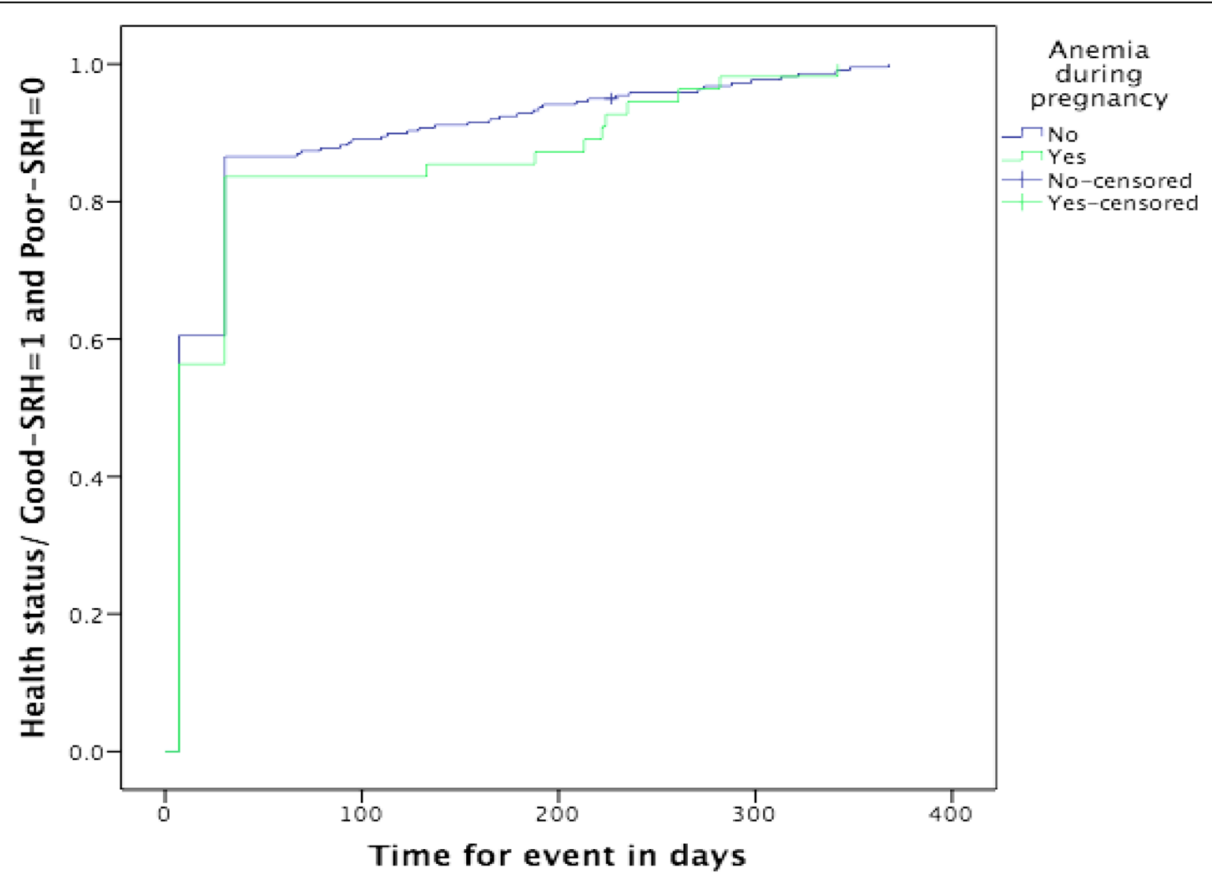

Fig. 4 Kaplan-Meier curve showing change of health status from poor-SRH to good-SRH by anemia during pregnancy, during follow-up time ${ }^{\mathrm{a}}\left(n=296^{\mathrm{b}}\right)$. aTime of interview is time end point for follow-up of each woman. ${ }^{\mathrm{b}}$ Only women reporting poor-SRH at day one are included in analysis 
Table 3 Good-SRH ${ }^{a}$ and poor-SRH ${ }^{b}$ in relation to background variables. Univariable logistic regression analysis ${ }^{\mathrm{c}}$ for poor-SRH in relation to background variables at three times ${ }^{\mathrm{d}}$

\begin{tabular}{|c|c|c|c|c|c|c|c|c|c|}
\hline & \multicolumn{3}{|c|}{ SRH at day one after delivery } & \multicolumn{3}{|c|}{ SRH at one month after delivery } & \multicolumn{3}{|c|}{$\mathrm{SRH}$ at the time of the interview } \\
\hline & Good-SRH & Poor-SRH & $\begin{array}{l}\text { Crude } O R \text { and its } \\
95 \% \mathrm{Cl}\end{array}$ & Good-SRH & Poor-SRH & $\begin{array}{l}\text { Crude } \mathrm{OR} \text { and its } \\
95 \% \mathrm{Cl}\end{array}$ & Good-SRH & Poor-SRH & $\begin{array}{l}\text { Crude } \mathrm{OR} \text { and its } \\
95 \% \mathrm{Cl}\end{array}$ \\
\hline \multicolumn{10}{|l|}{ Women's age (years) } \\
\hline$<25$ & $198(67.6 \%)$ & $97(32.4 \%)$ & $1.02(0.75-1.38)$ & $265(89.8 \%)$ & $30(10.2 \%)$ & $1.93(1.12-3.32)^{\mathrm{e}}$ & $263(89.8 \%)$ & $30(10.2 \%)$ & $0.82(0.51-1.31)$ \\
\hline $25-34$ & $329(67.1 \%)$ & $158(32.9 \%)$ & 1 & $461(94.5 \%)$ & $27(5.5 \%)$ & 1 & $427(87.9 \%)$ & $59(12.1 \%)$ & 1 \\
\hline$\geq 35$ & $96(70.6 \%)$ & $40(29.4 \%)$ & $0.86(0.57-1.31)$ & $122(89.7 \%)$ & $14(10.3 \%)$ & $1.95(0.99-3.85)$ & $118(86.8 \%)$ & $18(13.2 \%)$ & $1.10(0.62-1.94)$ \\
\hline \multicolumn{10}{|l|}{ Place of delivery } \\
\hline At health facility & $589(67.5 \%)$ & $283(32.5 \%)$ & 1 & $806(92.4 \%)$ & $66(7.6 \%)$ & 1 & $772(89.0 \%)$ & $95(11.0 \%)$ & 1 \\
\hline $\begin{array}{l}\text { At home or on the way to health } \\
\text { facility }\end{array}$ & $33(73.3 \%)$ & $12(26.7 \%)$ & $0.75(0.38-1.48)$ & $40(87.0 \%)$ & $6(13.0 \%)$ & $1.83(0.74-4.47)$ & $36(76.6 \%)$ & $11(23.4 \%)$ & $2.48(1.22-5.04)$ \\
\hline \multicolumn{10}{|l|}{ Mode of delivery } \\
\hline Vaginal (with or without instruments) & $569(71.1 \%)$ & $231(28.9 \%)$ & 1 & $746(93.1 \%)$ & $55(6.9 \%)$ & 1 & $699(87.7 \%)$ & $98(12.3 \%)$ & 1 \\
\hline Caesarean Section & $46(41.4 \%)$ & $65(58.6 \%)$ & $3.48(2.31-5.23)$ & $95(85.6 \%)$ & $16(14.4 \%)$ & $2.28(1.25-4.14)$ & $102(91.9 \%)$ & $9(8.1 \%)$ & $0.62(0.30-1.28)$ \\
\hline \multicolumn{10}{|l|}{$\begin{array}{l}\text { Hypertension during pregnancy and } \\
\text { delivery }\end{array}$} \\
\hline No & $602(69.2 \%)$ & $268(30.8 \%)$ & 1 & $808(92.8 \%)$ & $63(7.2 \%)$ & 1 & $767(88.5 \%)$ & $100(11.5 \%)$ & 1 \\
\hline Yes & $21(42.9 \%)$ & $28(57.1 \%)$ & $2.99(1.67-5.37)$ & $40(81.6 \%)$ & $9(18.4 \%)$ & $2.88(1.34-6.21)$ & $42(85.7 \%)$ & $7(14.3 \%)$ & $1.27(0.55-2.92)$ \\
\hline \multicolumn{10}{|l|}{$\begin{array}{l}\text { Convulsions during pregnancy, delivery } \\
\text { and post partum }\end{array}$} \\
\hline No & $604(68.3 \%)$ & $280(31.7 \%)$ & 1 & $819(92.5 \%)$ & $66(7.5 \%)$ & 1 & $782(88.7 \%)$ & $100(11.3 \%)$ & 1 \\
\hline Yes & $19(54.3 \%)$ & $16(45.7 \%)$ & $1.81(0.92-3.58)$ & $29(82.9 \%)$ & $6(17.1 \%)$ & $2.56(1.02-6.40)$ & $27(79.4 \%)$ & $7(20.6 \%)$ & $2.02(0.86-4.77)$ \\
\hline \multicolumn{10}{|l|}{ Diabetes mellitus during pregnancy } \\
\hline No & $608(67.6 \%)$ & $291(32.4 \%)$ & 1 & $828(92.0 \%)$ & $72(8.0 \%)$ & 1 & $791(88.3 \%)$ & $105(11.7 \%)$ & 1 \\
\hline Yes & $15(75.0 \%)$ & $5(25.0 \%)$ & $0.69(0.25-1.93)$ & $20(100.0 \%)$ & $0(0.0 \%)$ & 0.000 & $18(90.0 \%)$ & $2(10.0 \%)$ & $0.83(0.19-3.65)$ \\
\hline \multicolumn{10}{|l|}{ Anaemia during pregnancy } \\
\hline No & $542(69.4 \%)$ & $239(30.6 \%)$ & 1 & $729(93.2 \%)$ & $53(6.8 \%)$ & 1 & $711(91.4 \%)$ & $67(8.6 \%)$ & 1 \\
\hline Yes & $81(58.7 \%)$ & $57(41.3 \%)$ & $1.59(1.10-2.31)$ & $119(86.2 \%)$ & $19(13.8 \%)$ & $2.19(1.25-3.84)$ & $98(71.0 \%)$ & $40(29.0 \%)$ & $4.33(2.77-6.75)$ \\
\hline \multicolumn{10}{|l|}{ Marital status } \\
\hline Married or cohabiting & $514(66.6 \%)$ & $258(33.4 \%)$ & 1 & $712(92.1 \%)$ & $61(7.9 \%)$ & 1 & $687(89.2 \%)$ & $83(10.8 \%)$ & 1 \\
\hline $\begin{array}{l}\text { Unmarried or single or widow or } \\
\text { separated }\end{array}$ & $108(74.0 \%)$ & $38(26.0 \%)$ & $1.42(0.95-2.12)$ & 135 (92.5 \%) & $11(7.5 \%)$ & $1.05(0.53-2.05)$ & $121(83.4 \%)$ & $24(16.6 \%)$ & $0.60(0.37-0.99)$ \\
\hline \multicolumn{10}{|l|}{ Age at marriage (years) } \\
\hline$<20$ & $170(69.7 \%)$ & 74 (30.3\%) & 1 & $227(93.0 \%)$ & $17(7.0 \%)$ & 1 & $212(86.9 \%)$ & $32(13.1 \%)$ & 1 \\
\hline$\geq 20$ & 365 (65.4 \%) & 193 (34.6 \%) & $1.21(0.87-1.67)$ & 511 (91.4 \%) & $48(8.6 \%)$ & $1.25(0.70-2.22)$ & 496 (89.2 \%) & 60 (10.8 \%) & $0.81(0.50-1.26)$ \\
\hline
\end{tabular}


Table 3 Good-SRH ${ }^{a}$ and poor-SRH ${ }^{b}$ in relation to background variables. Univariable logistic regression analysis ${ }^{c}$ for poor-SRH in relation to background variables at three times ${ }^{d}$ (Continued)

\begin{tabular}{|c|c|c|c|c|c|c|c|c|c|}
\hline \multicolumn{10}{|l|}{ Number of births } \\
\hline 1 & $185(67.8 \%)$ & 88 (32.2 \%) & 1 & $253(92.7 \%)$ & $20(7.3 \%)$ & 1 & $248(91.2 \%)$ & $24(8.8 \%)$ & 1 \\
\hline $2-4$ & $258(64.7 \%)$ & $141(35.3 \%)$ & $1.14(0.82-1.59)$ & 368 (92.0 \%) & $32(8.0 \%)$ & $1.10(0.61-1.96)$ & $351(88.2 \%)$ & $47(11.8 \%)$ & $1.38(0.82-2.32)$ \\
\hline$>4$ & $160(74.4 \%)$ & $55(25.6 \%)$ & $0.72(0.48-1.07)$ & 197 (91.6\%) & $18(8.4 \%)$ & $1.15(0.59-2.24)$ & $182(84.7 \%)$ & $33(15.3 \%)$ & $1.87(1.07-3.27)$ \\
\hline \multicolumn{10}{|l|}{ Discharge time (days) } \\
\hline$<3$ & $345(72.8 \%)$ & $129(27.2 \%)$ & $N A^{f}$ & 435 (91.8 \%) & $39(8.2 \%)$ & 1 & $422(89.4 \%)$ & $50(10.6 \%)$ & 1 \\
\hline 3 & $189(69.2 \%)$ & $84(30.8 \%)$ & & $261(95.6 \%)$ & $12(4.4 \%)$ & $0.51(0.26-0.99)$ & $235(86.4 \%)$ & $37(13.6 \%)$ & $1.83(0.42-7.90)$ \\
\hline $4-7$ & $61(51.3 \%)$ & $58(48.7 \%)$ & & 109 (91.6\%) & $10(8.4 \%)$ & $1.02(0.49-2.11)$ & $104(88.9 \%)$ & $13(11.1 \%)$ & $2.44(0.56-10.62)$ \\
\hline$>7$ & $13(39.4 \%)$ & $20(60.6 \%)$ & & $25(75.8 \%)$ & $8(24.2 \%)$ & $3.56(1.50-8.44)$ & 31 (93.9\%) & $1(6.1 \%)$ & $1.93(0.41-9.05)$ \\
\hline \multicolumn{10}{|l|}{ Handicapping complication } \\
\hline No & $609(69.0 \%)$ & $274(31.0 \%)$ & 1 & 820 (92.8 \%) & $64(7.2 \%)$ & 1 & $782(88.9 \%)$ & $17(1.9 \%)$ & 1 \\
\hline Yes & 14 (38.9\%) & $22(61.1 \%)$ & $3.49(1.76-6.92)$ & $28(77.8 \%)$ & $8(22.2 \%)$ & $3.66(1.60-8.36)$ & $27(75.0 \%)$ & $9(25.0 \%)$ & $2.66(1.21-5.82)$ \\
\hline \multicolumn{10}{|l|}{ Health Insurance } \\
\hline Yes & $511(69.7 \%)$ & $222(30.3 \%)$ & 1 & $679(92.6 \%)$ & $54(7.4 \%)$ & 1 & $650(89.2 \%)$ & $79(10.8 \%)$ & 1 \\
\hline No & $112(60.2 \%)$ & $74(39.8 \%)$ & $1.52(1.09-2.12)$ & $168(90.4 \%)$ & $18(9.6 \%)$ & $1.33(0.76-2.34)$ & $159(85.0 \%)$ & $28(15.0 \%)$ & $1.44(0.91-2.30)$ \\
\hline \multicolumn{10}{|l|}{ Women's education } \\
\hline $\begin{array}{l}\text { Completed secondary level and } \\
\text { reached university level }\end{array}$ & $141(65.3 \%)$ & 75 (34.7\%) & 1 & $194(89.8 \%)$ & $22(10.2 \%)$ & 1 & $195(91.1 \%)$ & $19(8.9 \%)$ & 1 \\
\hline Completed primary level & $411(67.8 \%)$ & 195 (32.2 \%) & $1.29(0.80-2.09)$ & 565 (93.1 \%) & $42(6.9 \%)$ & $0.82(0.37-1.81)$ & $527(87.1 \%)$ & 78 (12.9\%) & $1.28(0.64-2.58)$ \\
\hline No education & 71 (73.2 \%) & $26(26.8 \%)$ & $1.45(0.85-2.46)$ & 89 (91.8\%) & $8(8.2 \%)$ & $1.26(0.54-2.94)$ & 87 (89.7 \%) & $10(10.3 \%)$ & $0.84(0.37-1.89)$ \\
\hline \multicolumn{10}{|l|}{ Woman occupation } \\
\hline Employed & 437 (67.3\%) & $212(32.7 \%)$ & 1 & $598(92.0 \%)$ & $52(8.0 \%)$ & 1 & $575(88.6 \%)$ & $74(11.4 \%)$ & 1 \\
\hline Non employed & $176(68.5 \%)$ & 81 (31.5\%) & $1.05(0.77-1.43)$ & $238(92.6 \%)$ & $19(7.4 \%)$ & $1.08(0.63-1.88)$ & $223(87.8 \%)$ & $31(12.2 \%)$ & $0.92(0.59-1.44)$ \\
\hline \multicolumn{10}{|l|}{ Infection during pregnancy } \\
\hline No & 610 (67.8 \%) & 290 (32.2 \%) & 1 & 836 (92.8 \%) & 65 (7.2\%) & 1 & 797 (88.9 \%) & $100(11.1 \%)$ & 1 \\
\hline Yes & $13(68.4 \%)$ & $6(31.6 \%)$ & $0.97(0.36-2.58)$ & $12(63.2 \%)$ & 7 (36.8 \%) & $7.50(2.85-19.70)$ & $12(63.2 \%)$ & 7 (36.8 \%) & $4.64(1.78-12.08)$ \\
\hline \multicolumn{10}{|l|}{ Alcohol } \\
\hline No & 450 (66.8 \%) & 224 (33.2 \%) & 1 & 616 (91.3 \%) & $59(8.7 \%)$ & 1 & $589(87.5 \%)$ & $84(12.5 \%)$ & 1 \\
\hline Yes & 172 (70.5 \%) & $72(29.5 \%)$ & $1.18(0.86-1.63)$ & 231 (94.7 \%) & $13(5.3 \%)$ & $1.70(0.91-3.16)$ & $220(90.5 \%)$ & $23(9.5 \%)$ & $1.36(0.83-2.21)$ \\
\hline \multicolumn{10}{|l|}{ Smoking } \\
\hline No & 605 (67.5 \%) & 291 (32.5 \%) & 1 & 826 (92.1%) & 71 (7.9\%) & 1 & $790(88.5 \%)$ & $103(11.5 \%)$ & 1 \\
\hline Yes & 18 (78.3 \%) & 5 (21.7 \%) & $0.57(0.21-1.57)$ & 22 (95.7\%) & 1 (4.3\%) & $0.52(0.07-3.98)$ & 19 (82.6 \%) & 4 (17.4 \%) & $1.61(0.53-4.83)$ \\
\hline
\end{tabular}


Table 3 Good-SRH and poor-SRH $H^{b}$ in relation to background variables. Univariable logistic regression analysis ${ }^{c}$ for poor-SRH in relation to background variables at three times ${ }^{d}$ (Continued)

\begin{tabular}{|c|c|c|c|c|c|c|c|c|c|}
\hline \multicolumn{10}{|c|}{$\begin{array}{l}\text { Severe bleeding during pregnancy } \\
\text { and labour }\end{array}$} \\
\hline No & $606(68.5 \%)$ & $279(31.5 \%)$ & 1 & $821(92.7 \%)$ & $65(7.3 \%)$ & 1 & $780(88.3 \%)$ & $103(11.7 \%)$ & 1 \\
\hline Yes & $17(50.0 \%)$ & $17(50.0 \%)$ & $2.17(1.09-4.31)$ & $27(79.4 \%)$ & $7(20.6 \%)$ & $3.27(1.37-7.80)$ & $29(87.9 \%)$ & $4(12.1 \%)$ & $1.04(0.36-3.03)$ \\
\hline \multicolumn{10}{|c|}{ Significant blood loss after delivery } \\
\hline No & $576(69.6 \%)$ & $252(30.4 \%)$ & 1 & 772 (93.1 \%) & $57(6.9 \%)$ & 1 & $737(89.3 \%)$ & $88(10.7 \%)$ & 1 \\
\hline Yes & $47(51.6 \%)$ & 44 (48.4 \%) & $2.14(1.38-3.31)$ & $76(83.5 \%)$ & $15(16.5 \%)$ & $2.67(1.44-4.94)$ & $72(79.1 \%)$ & $19(20.9 \%)$ & $2.21(1.27-3.83)$ \\
\hline \multicolumn{10}{|l|}{ Breast feeding } \\
\hline Yes & $N A^{f}$ & & & 837 (92.3 \%) & $70(7.7 \%)$ & 1 & $798(88.4 \%)$ & $105(11.6 \%)$ & 1 \\
\hline No & & & & $4(66.7 \%)$ & $2(33.3 \%)$ & $5.97(1.07-33.21)$ & $6(100.0 \%)$ & $0(0.0 \%)$ & 000000 \\
\hline \multicolumn{10}{|c|}{ Household income per month } \\
\hline$>35,000$ RWF & $253(69.7 \%)$ & $110(30.3 \%)$ & 1 & 339 (93.4 \%) & $24(6.6 \%)$ & 1 & $333(91.5 \%)$ & $31(8.5 \%)$ & 1 \\
\hline 17,500-35,000 RWF & $164(68.6 \%)$ & 75 (31.4 \%) & $1.05(0.73-1.49)$ & 219 (91.3 \%) & $21(8.8 \%)$ & $1.35(0.73-2.49)$ & $208(87.5 \%)$ & 30 (12.6\%) & $1.54(0.91-2.63)$ \\
\hline$<17,500$ RWF & $169(65.5 \%)$ & $89(34.5 \%)$ & $1.21(0.86-1.70)$ & 237 (91.9 \%) & $21(8.1 \%)$ & $1.25(0.68-2.30)$ & $218(85.5 \%)$ & $37(14.5 \%)$ & $1.82(1.09-3.02)$ \\
\hline
\end{tabular}

${ }^{\mathrm{a} G o o d-S R H}$ (Self-rated health status) includes very good and good health status categories

bPoor-SRH (Self-rated health status) includes very poor and poor health status categories

'Univariable logistic regression analysis with calculation of crude odds ratio (OR) and its $95 \%$ confidence interval (CI)

${ }^{\mathrm{d}}$ Three times post partum: one day, one month after delivery, and at the time of the interview

eStatistically significant odds ratios are in bold

fNA: Not applicable 
Table 4 Univariable and multivariable and logistic regression analysis ${ }^{\mathrm{a}}$ of poor-SRH ${ }^{\mathrm{b}}$ at three times post partum ${ }^{\mathrm{c}}$

\begin{tabular}{|c|c|c|c|c|c|c|}
\hline & \multicolumn{2}{|c|}{ Poor-SRH ${ }^{\mathrm{b}}$ at day one after delivery } & \multicolumn{2}{|c|}{ Poor-SRH at one month after delivery } & \multicolumn{2}{|c|}{ Poor-SRH at the time of the interview } \\
\hline & Univariable analysis & Multivariable analysis & Univariable analysis & Multivariable analysis & Univariable analysis & Multivariable analysis \\
\hline & $\begin{array}{l}\text { Crude Odds Ratios } \\
\text { and its } 95 \% \mathrm{Cl}\end{array}$ & $\begin{array}{l}\text { Adjusted }{ }^{d} \text { Odds Ratios } \\
\text { and its } 95 \% \mathrm{Cl}\end{array}$ & $\begin{array}{l}\text { Crude Odds Ratios } \\
\text { and its } 95 \% \mathrm{Cl}\end{array}$ & $\begin{array}{l}\text { Adjusted }{ }^{d} \text { Odds Ratios } \\
\text { and its } 95 \% \mathrm{Cl}\end{array}$ & $\begin{array}{l}\text { Crude Odds Ratios } \\
\text { and its } 95 \% \mathrm{Cl}\end{array}$ & $\begin{array}{l}\text { Adjusted } \text { d Odds Ratios } \text { and its } 95 \% \mathrm{Cl}\end{array}$ \\
\hline \multicolumn{7}{|l|}{ Women's age (years) } \\
\hline$<25$ & $1.02(0.75-1.38)$ & $1.07(0.72-1.60)$ & $1.93(1.12-3.32)^{\mathrm{e}}$ & $2.71(1.30-5.62)$ & $0.82(0.51-1.31)$ & $0.87(0.46-1.64)$ \\
\hline $25-34$ & 1 & 1 & 1 & 1 & 1 & 1 \\
\hline$>35$ & $0.86(0.57-1.31)$ & $0.94(0.56-1.58)$ & $1.95(0.99-3.85)$ & $1.44(0.61-3.40)$ & $1.10(0.62-1.94)$ & $0.77(0.37-1.57)$ \\
\hline \multicolumn{7}{|l|}{ Place of delivery } \\
\hline At health facility & 1 & & 1 & & 1 & 1 \\
\hline At home or on the way to health facility & $0.75(0.38-1.48)$ & & $1.83(0.74-4.47)$ & & $2.48(1.22-5.04)$ & $1.92(0.87-4.21)$ \\
\hline \multicolumn{7}{|l|}{ Mode of delivery } \\
\hline Vaginal (with or without instruments) & 1 & 1 & 1 & 1 & 1 & 1 \\
\hline Caesarean Section & $3.48(2.31-5.23)$ & $3.20(2.07-4.96)$ & $2.28(1.25-4.14)$ & $2.25(0.97-5.23)$ & $0.62(0.30-1.28)$ & $0.66(0.29-1.49)$ \\
\hline \multicolumn{7}{|l|}{ Hypertension during pregnancy and delivery } \\
\hline No & 1 & 1 & 1 & 1 & 1 & \\
\hline Yes & $2.99(1.67-5.37)$ & $2.38(1.22-4.62)$ & $2.88(1.34-6.21)$ & $1.30(0.47-3.55)$ & $1.27(0.55-2.92)$ & \\
\hline \multicolumn{7}{|c|}{ Convulsions during pregnancy, delivery and post partum } \\
\hline No & 1 & & 1 & 1 & 1 & \\
\hline Yes & $1.81(0.92-3.58)$ & & $2.56(1.02-6.40)$ & $0.76(0.21-2.67)$ & $2.02(0.86-4.77)$ & \\
\hline \multicolumn{7}{|l|}{ Diabetes mellitus during pregnancy } \\
\hline No & 1 & & 1 & & 1 & \\
\hline Yes & $0.69(0.25-1.93)$ & & 0.000 & & $0.83(0.19-3.65)$ & \\
\hline \multicolumn{7}{|l|}{ Anemia during pregnancy } \\
\hline No & 1 & 1 & 1 & 1 & 1 & 1 \\
\hline Yes & $1.59(1.10-2.31)$ & $1.34(0.88-2.01)$ & $2.19(1.25-3.84)$ & $2.37(1.25-4.49)$ & $4.33(2.77-6.75)$ & $4.45(2.69-7.35)$ \\
\hline \multicolumn{7}{|l|}{ Marital status } \\
\hline Married or cohabiting & 1 & 1 & 1 & 1 & 1 & 1 \\
\hline Unmarried or single or widow or separated & $1.42(0.95-2.12)$ & $1.60(1.02-2.51)$ & $1.05(0.53-2.05)$ & $1.51(0.66-3.47)$ & $0.60(0.37-0.99)$ & $0.49(0.26-0.93)$ \\
\hline \multicolumn{7}{|l|}{ Age at marriage (years) } \\
\hline$<20$ & 1 & & 1 & & 1 & \\
\hline$\geq 20$ & $1.21(0.87-1.67)$ & & $1.25(0.70-2.22)$ & & $0.81(0.50-1.26)$ & \\
\hline
\end{tabular}


Table 4 Univariable and multivariable and logistic regression analysis ${ }^{\mathrm{a}}$ of poor-SRH ${ }^{\mathrm{b}}$ at three times post partum ${ }^{\mathrm{c}}$ (Continued)

\begin{tabular}{|c|c|c|c|c|c|c|}
\hline \multicolumn{7}{|l|}{ Number of births } \\
\hline 1 & 1 & 1 & 1 & 1 & 1 & 1 \\
\hline $2-4$ & $1.14(0.82-1.59)$ & $1.19(0.80-1.76)$ & $1.10(0.61-1.96)$ & $1.55(0.74-3.22)$ & $1.38(0.82-2.32)$ & $1.71(0.89-3.29)$ \\
\hline$>4$ & $0.72(0.48-1.07)$ & $0.76(0.44-1.61)$ & $1.15(0.59-2.24)$ & $2.26(0.80-6.35)$ & $1.87(1.07-3.27)$ & $2.42(1.06-5.54)$ \\
\hline \multicolumn{7}{|l|}{ Time when discharged (days) } \\
\hline$<3$ & $N A^{f}$ & & 1 & 1 & 1 & \\
\hline 3 & & & $0.51(0.26-0.99)$ & $0.49(0.24-0.99)$ & $1.83(0.42-7.90)$ & \\
\hline $4-7$ & & & $1.02(0.49-2.11)$ & $0.64(0.25-1.64)$ & $2.44(0.56-10.62)$ & \\
\hline$>7$ & & & $3.56(1.50-8.44)$ & $2.47(0.83-7.30)$ & $1.93(0.41-9.05)$ & \\
\hline \multicolumn{7}{|l|}{ Handicapping complication } \\
\hline No & 1 & 1 & 1 & 1 & 1 & 1 \\
\hline Yes & $3.49(1.76-6.92)$ & $2.16(0.99-4.69)$ & $3.66(1.60-8.36)$ & $2.23(0.80-6.23)$ & $2.66(1.21-5.82)$ & $2.20(0.89-5.47)$ \\
\hline \multicolumn{7}{|l|}{ Health Insurance } \\
\hline Yes & 1 & 1 & 1 & & 1 & \\
\hline No & $1.52(1.09-2.12)$ & $1.59(1.11-2.28)$ & $1.33(0.76-2.34)$ & & $1.44(0.91-2.30)$ & \\
\hline \multicolumn{7}{|l|}{ Women's education } \\
\hline Completed secondary level or reached university level & 1 & & 1 & & 1 & \\
\hline Completed primary level & $1.29(0.80-2.09)$ & & $0.82(0.37-1.81)$ & & $1.28(0.64-2.58)$ & \\
\hline No education & $1.45(0.85-2.46)$ & & $1.26(0.54-2.94)$ & & $0.84(0.37-1.89)$ & \\
\hline \multicolumn{7}{|l|}{ Woman occupation } \\
\hline Employed & 1 & & 1 & & 1 & \\
\hline Non employed & $1.05(0.77-1.43)$ & & $1.08(0.63-1.88)$ & & $0.92(0.59-1.44)$ & \\
\hline \multicolumn{7}{|l|}{ Infection during pregnancy } \\
\hline No & 1 & & 1 & 1 & 1 & 1 \\
\hline Yes & $0.97(0.36-2.58)$ & & $7.50(2.85-19.70)$ & $6.94(2.14-22.53)$ & $4.64(1.78-12.08)$ & $3.73(1.20-11.59)$ \\
\hline \multicolumn{7}{|l|}{ Alcohol } \\
\hline No & 1 & & 1 & & 1 & \\
\hline Yes & $1.18(0.86-1.63)$ & & $1.70(0.91-3.16)$ & & $1.36(0.83-2.21)$ & \\
\hline \multicolumn{7}{|l|}{ Smoking } \\
\hline No & 1 & & 1 & & 1 & \\
\hline Yes & $0.57(0.21-1.57)$ & & $0.52(0.07-3.98)$ & & $1.61(0.53-4.83)$ & \\
\hline
\end{tabular}


Table 4 Univariable and multivariable and logistic regression analysis ${ }^{\mathrm{a}}$ of poor-SRH ${ }^{\mathrm{b}}$ at three times post partum ${ }^{\mathrm{c}}$ (Continued)

\begin{tabular}{|c|c|c|c|c|c|c|}
\hline \multicolumn{7}{|c|}{ Severe bleeding during pregnancy and labour } \\
\hline No & 1 & 1 & 1 & 1 & 1 & \\
\hline Yes & $2.17(1.09-4.31)$ & $1.74(0.77-3.92)$ & $3.27(1.37-7.80)$ & $2.96(1.09-8.02)$ & $1.04(0.36-3.03)$ & \\
\hline \multicolumn{7}{|c|}{ Significant blood loss after delivery } \\
\hline No & 1 & 1 & 1 & 1 & 1 & 1 \\
\hline Yes & $2.14(1.38-3.31)$ & $2.04(1.24-3.35)$ & $2.67(1.44-4.94)$ & $1.77(0.81-3.82)$ & $2.21(1.27-3.83)$ & $1.22(0.60-2.45)$ \\
\hline \multicolumn{7}{|l|}{ Breast feeding } \\
\hline Yes & $N A^{f}$ & & 1 & 1 & 1 & \\
\hline No & & & $5.97(1.07-33.21)$ & $9.54(1.50-60.39)$ & 0 & \\
\hline \multicolumn{7}{|c|}{ Household income per month } \\
\hline$>35,000$ RWF & 1 & & 1 & & 1 & 1 \\
\hline $17,500-35,000$ RWF & $1.05(0.73-1.49)$ & & $1.35(0.73-2.49)$ & & $1.54(0.91-2.63)$ & $1.47(0.83-2.62)$ \\
\hline$<17,500$ RWF & $1.21(0.86-1.70)$ & & $1.25(0.68-2.30)$ & & $1.82(1.09-3.02)$ & $1.65(0.94-2.87)$ \\
\hline
\end{tabular}

anivariable logistic regression analysis with calculation of crude odds ratio (OR) and its $95 \%$ confidence interval (CI)

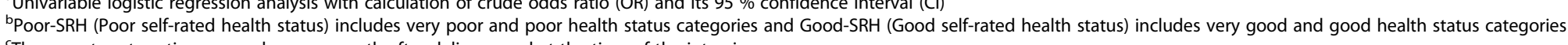

cThree post partum times: one day, one month after delivery, and at the time of the interview

${ }^{\mathrm{d} A d j u s t e d ~ O d d s ~ R a t i o s ~ f o r ~ w o m e n ' s ~ a g e, ~ n u m b e r ~ o f ~ b i r t h s, ~ m o d e ~ o f ~ d e l i v e r y, ~ a n d ~ m a r i t a l ~ s t a t u s ~}$

eStatistically significant odds ratios are in bold

fNA: Not applicable 
severe bleeding during pregnancy and labour $(\mathrm{OR}=3.60$, $\mathrm{CI}=1.56-8.31)$, hypertension during pregnancy and delivery $(\mathrm{OR}=2.21, \mathrm{CI}=1.06-4.60)$, significant postpartum haemorrage ( $\mathrm{OR}=2.01, \mathrm{CI}=1.12-3.58)$, woman's age less than 25 years $(\mathrm{OR}=1.71, \mathrm{CI}=1.05-2.80)$, and discharge time more than seven days postpartum $(\mathrm{OR}=2.81$, $\mathrm{CI}=1.18-6.66)$.

\section{Self-rated overall health status at one month postpartum}

In the univariable logistic regression, anaemia and infection during pregnancy, caesarean section, severe bleeding during pregnancy and labour, significant postpartum haemorrage, and handicapping complication (i.e., postpartum fistula or fecal and urinary incontinance) were associated with poor-SRH at one month postpartum (Table 3). In the multivariable logistic regression model, anaemia and infection during pregnancy, breastfeeding, severe bleeding during pregnancy and labour, and age less than 25 years were significantly associated with poor-SRH. Being discharged at third day postpartum was protective for reporting poor health at one month postpartum $(\mathrm{OR}=0.49, \mathrm{CI}=$ 0.24-0.99; ref.: discharge time before three days postpartum) (Table 4).

\section{Self-rated overall health status at time of interview}

For SRH at time of interview, the univariable logistic regression revealed an association with poor-SRH and place of delivery (i.e., at home or on the way to the health facility), anaemia and infection during pregnancy, handicapping complication (i.e., postpartum fistula or fecal and urinary incontinence), multiparity, and marital status (Table 3). In the multivariable analysis, multiparity was associated with poor-SRH at the time of interview (Table 4).

\section{Poor self-rated overall health at several times}

For SRH at one day and one week postpartum, the multivariable logistic regression model revealed an association with poor-SRH and caesarean section, hypertension during pregnancy and delivery, and significant postpartum haemorrage. Severe bleeding during pregnancy and labour was associated with poor-SRH at one week and one month postpartum. Infection and anaemia during pregnancy were associated with poor-SRH at one month postpartum $(\mathrm{OR}=$ $7.15, \mathrm{CI}=2.17-23.50$ and $\mathrm{OR}=2.32, \mathrm{CI}=1.22-4.38$, respectively) and at the time of the interview $(\mathrm{OR}=3.36$, $\mathrm{CI}=1.17-11.56)$ and $\mathrm{OR}=4.48, \mathrm{CI}=2.78-7.21$, respectively) (Table 4).

\section{Discussion}

This study found that approximately one-third of delivered women reported poor-SRH at one day postpartum. Participants' overall health status generally improved during the following year postpartum. Background factors such as marital status, age at marriage, education, occupation, smoking, and use of alcohol were not determinants of poor-SRH during the whole postpartum period. This study estimated the prevalence of poor-SRH to be $32.2 \%$ at one day, $7.8 \%$ at one month, and $11.2 \%$ at the time of the interview. The most plausible explanation of the increase of poor-SRH from one month postpartum to the time of interview is that women may have developed new health problems during their postpartum year. It is difficult to compare findings of this study with previous studies that have estimated poor health status at different times postpartum, as they have reported prevalences in the range of $4 \%$ to $15 \%$ between one and two years postpartum $[18,27,28]$. Nevertheless, we consider our poor-SRH prevalence at one day as comparatively high in the light of these previous studies.

Hypertension during pregnancy and delivery and significant postpartum haemorrhage were associated with poor-SRH at one day and one week postpartum, and severe bleeding during pregnancy and delivery were associated with poor-SRH at one week and one month postpartum. These findings are consistent with the literature that have identified these factors to be important determinants of poor-SRH during the early postpartum period $[27,29,30]$.

There are differing results on the impact of caesarian section on poor health status postpartum; in some studies poor health status at early postpartum is associated with caesarean section, [10,31], whereas others have not found any association [27, 32]. Our study showed caesarean section to be associated with poor-SRH at one day and one week after the operation.

The only sociodemographic factor that was associated with poor-SRH at one week and one month postpartum was age less than 25 years in relation to age 25 to 34 years. Similar results have also been shown in other studies [5, 33]; these results might be explained by the fact that young mothers are often more emotionally and physically vulnerable [5]. Low level of education was not found to be associated with poor-SRH in our study although other studies report it as a predictor of poor health status postpartum $[5,10]$. In Rwanda, utilization rate of maternal services before and during delivery is very high [23]. Both women with low and high levels of education have the same access to maternal health services including CHWs' support in the community, thus pregnant women's access is the same irrespective of educational levels [24, 34].

In this study, multiparity was associated with poor$\mathrm{SRH}$ at the time of the interview, a finding consistent with other studies that report multiparity to be 
associated with postpartum poor health status [15, 17]. Multiparity constitutes the potential risks of pregnancy and postpartum adverse outcomes [15].

Rwandan women who have experienced an uncomplicated delivery at a health facility are commonly discharged one to three days postpartum [23]. In our study, discharge on the third day postpartum in relation to discharge before three days was found to be protective of poor-SRH at one month postpartum. These results are coherent with the WHO recommendations to discharge women three days after giving birth [35]. Facility-based health care provided during the early postpartum period may have a positive effect on women's overall health status during the whole postpartum period.

Previous studies performed in low and middle income countries have found pregnancy-related factors such as anaemia and infection during pregnancy associated with health problems several months postpartum [27, 29]. In our study, the main determinants of postpartum poor$\mathrm{SRH}$ after one month were anaemia and infection during pregnancy.

\section{Methodological considerations}

One strength of this study is that almost all households and women selected were reached and consented to participate in the study. We consider the sample to be representative of the population under study. Another strength of this study was that female nurses, midwives, and clinical psychologists were used as interviewers in order to make female participants confident enough to respond. The representativity of this study undertaken in the Northern Province and Kigali is also supported by the fact that the data obtained for prevalence rates of ANC use, delivery at health facility, caesarean section, and postpartum care attendance were very similar to data available on maternal services delivery and use in Rwanda in the latest published Demographic Health Survey (DHS) 2014-15 results [23]. Recall bias may have been an issue since data were collected retrospectively, although previous studies have shown that recall bias is not a major issue when women are recalling their reproductive history [36, 37]. Another issue is that there may be underreporting or overreporting of some health conditions. In this study, the average time between the date of the interview and the date of the latest delivery was 7.1 months with a range of 1.4-14.3 months. This means that the time of interview for all participants corresponds to a wide time interval. Therefore, the results are influenced by the fact that some participants were interviewed at an early time point during the postpartum period when the health problems were still prevalent, whereas other participants were interviewed at a later time point when their health problems could have been resolved.

\section{Conclusions}

This study reports a high prevalence of poor SRH among Rwandan women in the early postpartum period. Infection, anaemia, hypertension, and severe bleeding during pregnancy and significant postpartum haemorrhage were the main determinants of poor-SRH during postpartum. Discharge time at third day postpartum in relation to discharge before three days postpartum decreased the risk of reporting poor-SRH at a later time. These incriminated determinants of poor-SRH are pregnancy and delivery-related factors, factors that may be prevented or reduced by more frequent and particular attention during pregnancy. Timely measures should be undertaken to deal with complications during delivery and early postpartum. The simple SRH status measurement can be used to identify determinants of poor health status. Further research is warranted to determine whether SRH inquiry may be used during antenatal care and postnatal care visits to screen for health problems and pregnancy complications related to poor health status postpartum.

\section{Acknowledgements \\ Our sincere thanks go to the participating Rwandan women. We also thank Anni-Maria Pulkki-Brännström and Anna Dencker for their helpful contribution to this study. We also thank the Swedish International Development Agency (SIDA) for funding, the University of Rwanda for logistic support, and Umeå University for overall support.}

\section{Funding}

This study was conducted as part of first author's PhD studies which was sponsored by the Swedish International Development Agency (SIDA). SIDA didn't play any role in the study design, data collection, data analysis and interpretation of data or in the writing of the manuscript.

\section{Availability of data and materials \\ The datasets used and analyzed during the current study will be available from the corresponding author upon receiving a reasonable request.}

\section{Authors' contributions}

All authors participated in the design of the study and the construction of the study tools. All authors contributed to the drafting of the manuscript. JPSS participated in data collection in the field in collaboration with MN and CM. JPSS performed the data analysis under supervision of IM. JPSS wrote the manuscript under supervision of $\mathrm{IM}$ and in collaboration with all other authors. All authors read and approved the final version of the manuscript.

\section{Competing of interests}

The authors declare that they have no competing interests.

\section{Consent for publication}

Not applicable.

\section{Ethics approval and consent to participate}

The research protocol and the study questionnaire were approved by University of Rwanda, College of Medicine and Health Sciences Institutional Review Board (Ref: 010/UR/CMHS/SPH/2014). The WHO guidelines on ethical issues were followed and participants were informed about their freedom to participate and to withdraw from the study at any time they wanted [38]. Before the interviews, interviewers provided information about the study to the participants. All selected participants signed a written consent form before their participation in the study. Before data collection, some health facilities were contacted in order to be prepared to take care of women still experiencing health problems related to their pregnancy and childbirth. 


\section{Author details}

1Department of Clinical Sciences, Obstetrics and Gynecology, Umeå University, Umeå, Sweden. ${ }^{2}$ University of Rwanda College of Medicine and Health Sciences School of Public Health, Kigali, Rwanda. ${ }^{3}$ Department of Community Medicine and Public Health, Sahlgrenska Academy, University of Gothenburg, Gothenburg, Sweden. ${ }^{4} J u d i t h$ Lumley Centre, La Trobe University, Melbourne, Australia.

\section{Received: 18 February 2016 Accepted: 30 October 2016}

Published online: 07 November 2016

\section{References}

1. Fayers PM, Sprangers MA. Understanding self-rated health. Lancet. 2002; 359(9302):187-18.

2. Chandola T, Jenkinson C. Validating self-rated health in different ethnic groups. Ethn Health. 2000;5(2):151-9.

3. Idler $E$, Angel R. Self-rated health and mortality in the NHANES- 1 epidemiologic follow-up. Am J Public Health. 1998;80(4):446-52.

4. Miilunpalo S, Vuori I, Oja P, Pasanen M, Urponen H. Self-rated health status as health measure: the predictive valua of self-reported health status on the use of physician services and on mortality in the working-age population. J Clin Epidemiol. 1997:50(5):517-28.

5. Schytt $E$, Waldenstrom $U$. Risk factors for poor self-rated health in women at 2 months and 1 year after childbirth. J Womens Health. 2007:16(3):390-405.

6. Fottrell E, Kanhonou L, Goufodji S, Behague DP, Marshall T, Patel V, Filippi V. Risk of psychological distress following severe obstetric complications in Benin: the role of economics, physical health and spousal abuse. Br J Psychiatry. 2010; 196(1):18-25.

7. Sedgh G, Singh S, Hussain R. Intended and unintended pregnancies worldwide in 2012 and recent trends. Stud Fam Plann. 2014;45(3):301-14.

8. Khan KS, Wojdyla D, Say L, Gulmezoglu AM, Van Look PF. WHO analysis of causes of maternal death: a systematic review. Lancet. 2006;367(9516):1066-74.

9. Albers LL. Health problems after childbirth. J Midwifery Womens Health. 2000;45(1):55-7.

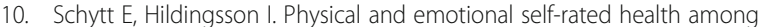
Swedish women and men during pregnancy and the first year of parenthood. Sex Reprod Healthc. 2011;2:57-64.

11. Gjerdinen D, Froberg D, Chaloner K, McGovern P. Changes in women's physical health during the first postpartum year. Arch Fam Med. 1993;2(3):277-83.

12. Waterstone $M$, Wolfe $C$, Hooper $R$, Bewley S. Postnatal morbidity after childbirth and severe obstetric morbidity. BJOG. 2003;110(2):128-33.

13. Saurel-Cubizolles MJ, Romito P, Lelong N, Ancel PY. Women's health after childbirth: a longitudinal study in France and Italy. Br J Obstet Gynaecol. 2000;107:1202-9.

14. Mohan D, Gupta S, Lefevre A, Bazant E, Killewo J, Baqui HA. Determinants of postnatal care use at health facilities in rural Tanzania: multilevel analysis of a household survey. BMC Pregnancy Childbirth. 2015:15:282.

15. Alsammani AM, Ahmed SR. Grand multiparity: risk factors and outcome in a tertiary hospital: a comparative study. Mater Sociomed. 2015;27(4):244-7.

16. Webb D, Bloch J, Coyne J, Chung E, Bennet I, Culhane J: Postpartum Physical Symptoms in New Mothers: Their Relationship to Functional Limitations and Emotional Well-being. Birth 2008;35(3). doi:10.1111/j.1523536X.2008.00238.x

17. Henderson J, Redshaw M: Who is Well After Childbirth? Factors Related to Positive Outcome. BIRTH 2013, 40(1):1-9.

18. Morgan KJ, Eastwood JG: Social determinants of maternal self-rated health in South Western Sydney, Australia. BMC Research. 2014;7(51). doi:10.1186/ 1756-0500-7-51.

19. WHO. Rwanda maternal and perinatal country profile. 2014:40(1):1-9.

20. Health RMo. Rwanda annual health statistics booklet 2014. 2015

21. Condo J, Mugeni C, Naughton B, Hall K, Tuazon MA, Omwega A, Nwaigwe F, Drobac P, Hyder Z, Ngabo F, et al. Rwanda's evolving community health worker system: a qualitative assessment of client and provider perspectives. Hum Resour Health. 2014;12:71

22. (NISR) NloSoR, $(\mathrm{MOH}) \mathrm{RMoH}$, International I. Rwanda demographic and health survey 2010. 2012

23. Rwanda NloSo, $(\mathrm{MOH}) \mathrm{RMoH}$, International I. Rwanda demographic and health survey 2015. 2015.

24. Health RMo. National community health policy 2008. 2008.
25. Nathan LM, Shi Q, Zhang C, Nsabimana D, Skalr M, Mutimura E, Merkatz IR, Einstein MH, Anastos K. Decentralizing maternal services to increase skilled attendance at birth and antenatal care utilization in rural rwanda: a prospective cohort study. Matern Child Health J. 2015; 19:1945-55.

26. National Institute of Statistics of Rwanda (NISR) MoFaEPMR. Fourth rwanda population and housing census. 2012.

27. Kabakian-Khasholian T, Shayboub R, Ataya A. Health after childbirth: patterns of reported postpartum morbidity from Lebanon. Women Birth. 2014;27(1):15-20.

28. Molarius A, Berglund K, Eriksson C, Lambe M, Nordstrom E, Eriksson HG, Feldman I. Socioeconomic conditions, lifestyle factors, and self-rated health among men and women in Sweden. Eur J Public Health. 2007; 17(2):125-33.

29. Brown S, Lumley J. Maternal health after childbirth: results of an Australian population based survey. Br J Obstet Gynaecol. 1998;105(2):156-61.

30. Seck SM, Diop-Dia A, Dia DG, Gueye L. Prevalence of hypertension and assessment of its impact on self-rated health in rural populations: a crosssectional study in northern Senegal. Med Sante Trop. 2015;25(2):160-4.

31. Rowlands IJ, Redshaw M. Mode of birth and women's psychological and physical wellbeing in the postnatal period. BMC Pregnancy Childbirth. 2012:12:138.

32. Borders N. After the afterbirth: a critical review of postpartum health relative to method of delivery. J Midwifery Womens Health. 2006:51(4):242-8.

33. Moum T. Self-assessed health among Norwegian adults. Soc Sci Med. 1992; 35(7):935-47.

34. Manzi A, Munyaneza F, Mujawase F, Banamwana L, Sayinzoga F, Thomson DR, Ntaganira J, Hedt-Gauthier BL. Assessing predictors of delayed antenatal care visits in Rwanda: a secondary analysis of Rwanda demographic and health survey 2010. BMC Pregnancy Childbirth. 2014;14:290.

35. WHO. Who technical consultation on postpartum and postnatal care. 2010.

36. Radin RG, Rothman K, Hatch EE, Mikkelsen EM, Sorensen HT, Riis AH, Fox MP. Wise LA. Maternal recall error in retrospectively reported time-topregnancy: an assessment and bias analysis. Paediatr Perinat Epidemiol. 2015;29(6):576-88

37. Casey VA, Dwyer JT, Coleman KA, Krall EA, Gardner J, Valadian I. Accuracy of recall by middle-aged participants in a longitudinal study of their body size and indices of maturation earlier in life. Ann Hum Biol. 1991;18(2):155-66.

38. WHO. Putting women first: ethical and safety. Recommendations for research on domestic violence against women. Geneva: Global program on evidence for health policy; 1999.

\section{Submit your next manuscript to BioMed Central and we will help you at every step:}

- We accept pre-submission inquiries

- Our selector tool helps you to find the most relevant journal

- We provide round the clock customer support

- Convenient online submission

- Thorough peer review

- Inclusion in PubMed and all major indexing services

- Maximum visibility for your research

Submit your manuscript at www.biomedcentral.com/submit 